\title{
BRIGHTNESS ENHANCEMENT LIMITS IN Cladding-PUMPED FibER RAMAN AMPLIFIERS
}

\author{
Junhua Ji, Christophe A. Codemard, and Johan Nilsson \\ Optoelectronics Research Centre, University of Southampton, SO17 1BJ, Southampton UK.
}

\begin{abstract}
We analyze theoretically limitations on the brightness enhancement of a multimode pump beam, to be efficiently converted into a diffraction-limited Stokes beam in a cladding-pumped fiber Raman amplifier. For a given minimum Raman pump absorption, parasitic $2^{\text {nd }}$ Stokes generation limits the cladding-to-core area ratio, and thus the brightness enhancement. A W-type fiber acting as a spectral waveguide filter allows for nearly five times larger inner-cladding areas by suppressing the $2^{\text {nd }}$ Stokes. We further analyze limits set by glass damage and indirectly propagation loss, as well as pulse walk-off. A well-designed fiber with $3.5 \mathrm{~dB} / \mathrm{km}$ propagation loss allows for a pump-to-signal brightness improvement of up to 3600 times both in the pulsed and the $\mathrm{cw}$ regime.
\end{abstract}

\section{INTRODUCTION}

In recent years we have demonstrated and analyzed $\mathrm{cw}$ and pulsed cladding pumped fiber Raman amplification (CPFRA) at $1.66 \mu \mathrm{m}$ and $1.1 \mu \mathrm{m}[1-5]$. In this novel approach, a low-brightness pump which is propagating in a passive double-clad fiber is converted through stimulated Raman scattering (SRS) into a diffraction-limited core mode at the Stokes wavelength. The operation of CPFRAs is similar to that of rare-earth doped double-clad fibers, with the advantage that this scheme is wavelength-flexible, limited only by the transparency range of the fiber. Other advantages are that it is possible to reach very high gain in the pulsed regime $[2,4]$ without being limited by ASE since there is no energy storage involved in the amplification process, and a low relative quantum defect, in particular at shorter wavelengths.

\section{LIMITATIONS ON BRIGHTNESS ENHANCEMENT}

We have shown in [2] the design requirements for efficient power conversion in a cladding-pumped Raman fiber amplifier, in a basic double clad fiber design with step-index core and cladding. We showed that a 2 $\mathrm{Np}(=8.9 \mathrm{~dB})$ pump depletion by the signal as required for efficient operation imposes a restriction on the cladding-to-core area ratio of approximately $\sim 8$. Otherwise, the $2^{\text {nd }}$ Stokes will inevitably build up, which depletes the power in the $1^{\text {st }}$ Stokes and limits the brightness enhancement (BE) process. This is a first limitation. However, more sophisticated fiber designs allow for higher BE whilst maintaining high conversion efficiency. We have found that with a W-type core [6] design, it is possible to achieve a cladding-to-core area ratio (CCAR) of about 34 with large core areas. This design is such that the SRS from the $1^{\text {st }}$ to the $2^{\text {nd }}$ Stokes is reduced, allowing for a larger cladding and therefore relaxing the limit on the area ratio set by the $2^{\text {nd }}$ Stokes.

A second limitation on the CCAR is set by optical damage in the core. A high pump intensity combined with a large CCAR may well lead to $1^{\text {st }}$ Stokes intensities in the core that exceed the damage threshold. The area ratio limitation from material damage will depend on the pulse duration because of its influence on the damage threshold. Here, we assume that the damage intensity is proportional to the inverse of the square-root of the pulse duration [7], and reaches the cw damage threshold for a duration of $625 \mathrm{~ns}$. Thus, data for $625 \mathrm{~ns}$ pulses are valid for the $\mathrm{cw}$ regime. Note also that although a lower pump intensity allows for a higher degree of $\mathrm{BE}$, the propagation loss and thus the useable fiber length limits the use of low pump intensities.

Finally, we find that the pulse walk-off that takes place in any multimode fiber limits the BE, too. In a CPRFA, this is important since the energy is directly transferred from the pump to the Stokes waves, so these must overlap temporally for efficient operation. In addition, the pump and signal pulses can experience temporal broadening. These effects limit the interaction length over which Raman amplification occurs. Specifically, we find that pulse walk-off limits the cladding NA and the fiber length, which also in this case is related to the pump intensity. 
We can combine these limitations to determine the maximum $\mathrm{BE}$ that is possible to achieve in a CPRFA. Figure 1 and 2 show the maximum BE that can be reached for various pump intensities in a double-clad fiber with an all-glass outer cladding and for a jacketed air-clad fiber. Here, the pump and Stokes wavelength are $1.55 \mu \mathrm{m}$ and $1.66 \mu \mathrm{m}$; the damage threshold is assumed to be $500 \mathrm{~W} / \mu \mathrm{m}^{2}$ for a $1 \mathrm{~ns}$ pulse with an inverse square-root dependence of the damage threshold on the pulse duration. The core radius is fixed at $9 \mu \mathrm{m}$, which allows for a cladding-to-core area ratio of 34 in an optimized W-type fiber.

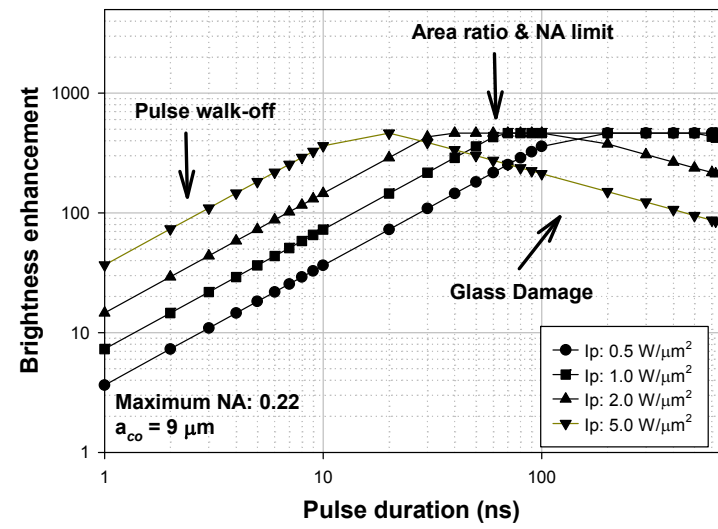

Fig. 1: Brightness enhancement limits in a claddingpumped solid all-glass fiber.

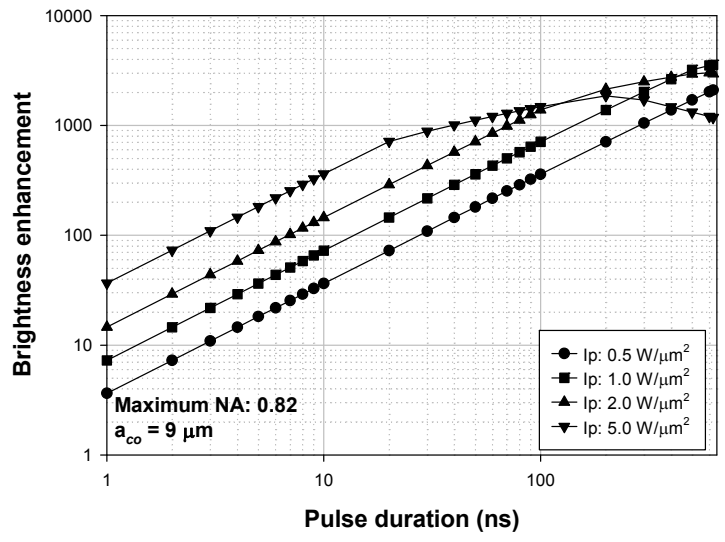

Fig. 2: Brightness enhancement limits in a cladding-pumped jacketed air-clad fiber.

In Fig. 1, in a fiber with a 0.22 inner cladding NA, the BE is limited by the pulse walk-off for short pump pulses, then the 2nd Stokes and the NA of the fiber limit any further BE for longer pulses. For even longer pulses, the glass damage reduces the BE that can be achieved. By contrast, with a high-NA inner cladding such as in the jacketed air-clad fiber, the BE is not limited by the inner cladding NA as shown in Fig. 2. In this case, a BE factor of $\sim 3600$ is possible. The highest BE-factor can be achieved with pump pulses longer than $100 \mathrm{~ns}$, as well as $\mathrm{cw}$. In the all-glass fiber, the maximum brightness enhancement is constant, $\sim 500$ over a range of pulse durations.

\section{CONCLUSIONS}

We have shown that core damage and pump pulse walk-off can limit the brightness enhancement in cladding-pumped Raman fiber. An analytical expression of the optimum pump pulse parameters will be given in the manuscript. For an air-clad fiber, the brightness enhancement can be about 400 to 3600 for pump pulses longer than $100 \mathrm{~ns}$.

\section{REFERENCE}

[1] J. Nilsson, J. K. Sahu, J. N. Jang, R. Selvas, D. C. Hanna, and A. B. Grudinin, "Cladding-pumped Raman fiber amplifier", in Proc. Topical Meeting on Optical Amplifiers and Their Applications, post-deadline paper PDP2, Vancouver, Canada, July 14 17,2002

[2] J. Ji, C. Codemard, M. Ibsen, J. K. Sahu, and J. Nilsson, "Analysis of the conversion to the first Stokes in cladding-pumped fiber Raman amplifiers," IEEE J. Sel. Top. Quantum Electron. 15, 29-139 (2009).

[3] C. A. Codemard, P. Dupriez, Y. Jeong, J. K. Sahu, M. Ibsen, and J. Nilsson, "High-power continuous-wave cladding-pumped Raman fiber laser," Opt. Lett. 31, 2290-2292 (2006).

[4] C. A. Codemard, J. K. Sahu, and J. Nilsson, "Cladding pumped Raman fiber amplifier for high-gain, high energy single-stage amplification," in "Optical Fiber Communication Conference and Exposition and The National Fiber Optic Engineers Conference," (Optical Society of America, 2005), p. OTuF5.

[5] A. Shirakawa, C. A. Codemard, J. Ji, K. K. Chen, A. Malinowski, D. J. Richardson, J. K. Sahu, and J. Nilsson, "High-brightness $210 \mu \mathrm{J}$ pulsed Raman fiber source," in "Conference on Lasers and Electro-Optics/Quantum Electronics and Laser Science Conference and Photonic Applications Systems Technologies," (Optical Society of America, 2008), p. CTuL1.

[6] J. Kim, P. Dupriez, C. Codemard, J. Nilsson, and J. K. Sahu, "Suppression of stimulated Raman scattering in a high power Ybdoped fiber amplifier using a W-type core with fundamental mode cut-off," Opt. Express 14, 5103-5113 (2006)

[7] B. C. Stuart, M. D. Feit, S. Herman, A. M. Rubenchik, B. W. Shore, and M. D. Perry, "Nanosecond-to-femtosecond laserinduced breakdown in dielectrics," Phys. Rev. B, 53, 1749-1761, 1996. 